\title{
SOME RESULTS ON ORIENTATION PRESERVING INVOLUTIONS
}

\author{
BY
}

DAVID E. GIBBS

\begin{abstract}
The bordism of orientation preserving differentiable involutions is studied by use of the signature-like invariant ab: $0_{*}\left(Z_{2}\right) \rightarrow$ $W_{0}\left(Z_{2} ; Z\right)$. The equivariant Witt ring $W_{0}\left(Z_{2} ; Z\right)$ is calculated and is shown to be isomorphic under ab to the effective part of $\mathrm{O}_{4}\left(Z_{2}\right)$. Modulo 2 relations are established between the representation of the involution on $H^{2 k}\left(M^{4 k} ; Z\right) /$ torsion and $X_{0}(F)$ and $X_{2}(F)$, where $x_{i}(F)$ is the Euler characteristic of those components of the fixed point set with dimensions congruent to $i$ modulo 4. For manifolds of dimension $4 k+2$, it is shown that $\chi_{0}(F) \equiv \chi_{2}(F) \equiv 0(\bmod 2)$. Finally the ideal $E_{0}\left(Z_{2} ; Z\right)$ consisting of those elements of $W_{0}\left(Z_{2} ; Z\right)$ admitting a representative of type $I$ is determined.
\end{abstract}

In this paper we give some results arrived at in studying the relation between $W_{0}\left(Z_{2} ; Z\right)$ and the bordism ring of orientation preserving differentiable involutions $O_{*}\left(Z_{2}\right)$.

The paper takes the following form. The equivariant Witt ring $W_{0}\left(Z_{2} ; Z\right)$ is defined and its relevant facts are discussed in $\$ 1$. In $\$ 2$ we define the AtiyahBott homomorphism ab: $O_{4 k}\left(Z_{2}\right) \rightarrow W_{0}\left(Z_{2} ; Z\right)$. This homomorphism plays a role in the study of $O_{*}\left(Z_{2}\right)$ analogous to the role the signature plays in studying closed oriented manifolds. Consistent with this analogy, we show that ab completely determines $\mathrm{O}_{4}\left(Z_{2}\right)$. To do so, we observe that $\mathrm{O}_{4}\left(Z_{2}\right) \cong \Omega_{4} \oplus \mathrm{O}_{4}\left(Z_{2}\right)^{e}$ in a natural way where $\mathrm{O}_{4}\left(Z_{2}\right)^{e}$ consists of those actions of $Z_{2}$ which are effective. Since $\Omega_{4}$ is determined by characteristic numbers, the problem is reduced to determining $\mathrm{O}_{4}\left(Z_{2}\right)^{e}$. We then prove that ab: $\mathrm{O}_{4}\left(Z_{2}\right)^{e} \rightarrow W_{0}\left(Z_{2} ; Z\right)$ is an isomorphism. Finally, in this section we note that if $\left(T, M^{4 k}\right)$ has $\chi_{2}(F) \equiv$ $0(\bmod 2)$ then there is some $\left(T_{1}, N^{4 k}\right)$ with no unoriented components in its fixed point set and $\mathrm{ab}\left(T, M^{4 k}\right)=\mathrm{ab}\left(T_{1}, N^{4 k}\right)$. In particular, if $\left(T, M^{4}\right)$ has $\chi_{2}(F) \equiv 0(\bmod 2)$ then it is bordant to a manifold with involution having no unoriented components in its fixed point set.

$\S 3$ establishes a relation between the global invariant $H^{2 k}\left(M^{4 k} ; Z\right) /$ tor

Received by the editors November 25, 1974 and, in revised form, May 12, 1975. AMS (MOS) subject classifications (1970). Primary 57D85; Secondary 10C05, $20 \mathrm{C10}$.

Key words and phrases. Equivariant bordism, Euler characteristic, orientation preserving involution, representation, Witt ring. 
and $\chi_{0}(F), \chi_{2}(F)$, and trs, where $\chi_{i}(F)$ is the Euler characteristic of those components of $F$ with dimensions congruent to $i$ modulo 4 and trs will be defined in $\$ 1$. Since we demand that the action of $Z_{2}$ preserve the orientation of $M^{4 k}$, it follows that $F$ has components in even dimensions only. To give the relation, we observe that the representation of $Z_{2}$ on a free finitely generated $Z$-module $V$ decomposes into indecomposable summands as

$$
V \cong Z \oplus \ldots \oplus Z \oplus Z\left(Z_{2}\right) \oplus \ldots \oplus Z\left(Z_{2}\right) \oplus \widetilde{Z} \oplus \ldots \oplus \widetilde{Z}
$$

where the $Z$ summands are fixed under $Z_{2}$, the action of $Z_{2}$ takes 1 to -1 in the $\widetilde{Z}$ summands, and $Z\left(Z_{2}\right)$ has rank 2 with the action interchanging the generators. Now we decompose $H^{2 k}\left(M^{4 k} ; Z\right)$ /tor with the $Z_{2}$ action given by $T^{*}$ and prove that if $r$ is the number of $Z$ summands, $s$ the number of copies of $Z\left(Z_{2}\right)$, and $t$ the number of $\widetilde{Z}$ summands, then $\chi_{0}(F) \equiv r(\bmod 2), \chi_{2}(F) \equiv t$ (mod 2), and trs is given by reading $s$ modulo 2 .

In $\S 4$, we prove that for a closed oriented $(4 n+2)$-manifold with differentiable involution the numbers $\chi_{0}(F)$ and $\chi_{2}(F)$ are even. It was previously known that $\chi_{0}(F)+\chi_{2}(F) \equiv 0(\bmod 2)$ but the fact that $\chi_{0}(F) \equiv \chi_{2}(F) \equiv 0$ $(\bmod 2)$ was unexpected.

In $\S 5$, we determine the ideal $E_{0}\left(Z_{2} ; Z\right)$ which consists of those elements of $W_{0}\left(Z_{2} ; Z\right)$ admitting a representative of type II. Our reason for doing so is the frequency (cf. [10]) with which highly connected manifolds give rise to inner product spaces (on their middle dimensional cohomology) of type II. Theorem 14 specifies generators for $E_{0}\left(Z_{2} ; Z\right)$ and we show as a corollary that

$$
W_{0}\left(Z_{2} ; Z\right) / E_{0}\left(Z_{2} ; Z\right) \cong Z_{8} \oplus Z_{2}
$$

where the summands are given by the signature of the form modulo 8 and $q(T, V)$. The multiplicative structure is given by

$$
\begin{aligned}
(\operatorname{sgn} V(\bmod 8), q(T, V)) & \cdot\left(\operatorname{sgn} V_{1}(\bmod 8), q\left(T_{1}, V_{1}\right)\right) \\
=(\operatorname{sgn} V & \operatorname{sgn} V_{1}(\bmod 8), \operatorname{sgn} V \operatorname{sgn} V_{1} \\
& \left.+\operatorname{sgn} V q\left(T_{1}, V_{1}\right)+\operatorname{sgn} V_{1} q(T, V)\right) .
\end{aligned}
$$

At this point, I would like to thank Pierre Conner for bringing this technique of studying transformation groups to my attention and for numerous helpful conversations.

1. In this section, we develop the requisite algebra. The results in this section are due to Pierre Conner.

As a first step, we recall from [11] that $W\left(Z_{2}\right) \cong Z_{2}$ and note that the exact sequence $[11$, p. 88$]$ can be modified in an obvious way to give a split exact sequence $0 \rightarrow Z \stackrel{i}{\longrightarrow} W(Z(1 / 2)) \stackrel{\partial}{\longrightarrow} W\left(Z_{2}\right) \rightarrow 0$, where $\partial$ is defined in [11]. Thus $W(Z(1 / 2)) \cong Z \oplus Z_{2}$. 
We now define $W_{0}\left(Z_{2} ; Z\right)$ by considering all pairs $\left(Z_{2}, V\right)$ where

(1) $V$ is a finitely generated free $Z$-module with a symmetric, bilinear and $Z$-valued inner product in the sense of [11], and

(2) $Z_{2}$ acts as a group of $Z$-module isometries. We say $\left(Z_{2}, V\right) \sim 0$ if there is a $Z_{2}$-invariant submodule $W \subset V$ such that $W$ is equal to its orthogonal complement $W^{\perp}$. Furthermore, we say that $\left(Z_{2}, V\right) \sim\left(Z_{2}, V^{\prime}\right)$ if $\left(Z_{2}, V\right) \oplus$ $\left(Z_{2},-V^{\prime}\right) \sim 0$. The Witt class of $\left(Z_{2}, V\right)$ will be denoted by $\left\langle Z_{2}, V\right\rangle$ and the collection of all such classes by $W_{0}\left(Z_{2} ; Z\right)$. Of course, $W_{0}\left(Z_{2} ; Z\right)$ is given the structure of a ring in the usual way.

In the above definition, it should be noted that the action of $Z_{2}$ is not required to be effective. It may be further observed with a bit of effort that the definition given here is the correct extension of the definition found in [11] to the case where there is a group action on the module. The stability property is obviated for $Z$-modules because, if an inner product space over $Z$ with involution stably splits, then the module itself splits.

Before we state the first result, we need to define a homomorphism

$$
i: W_{0}\left(Z_{2} ; Z\right) \rightarrow W(Z(1 / 2)) \oplus W(Z(1 / 2)) \text {. }
$$

To do so, we take an element $(T, V)$ and form $I_{+}=$the elements of $V$ fixed under $T$ and $I_{-}=$kernel $\Sigma$, where $\Sigma=\mathrm{Id}+T$. The homomorphism $i$ is now given by

$$
(T, V) \mapsto\left(\left[I_{+} \otimes Z(1 / 2)\right],\left[I_{-} \otimes Z(1 / 2)\right]\right)
$$

and is rather easily seen to be well defined and a monomorphism.

Proposition 1. The sequence

$$
0 \rightarrow W_{0}\left(Z_{2} ; Z\right) \stackrel{i}{\longrightarrow} W(Z(1 / 2)) \oplus W(Z(1 / 2)) \stackrel{\partial+\partial}{\longrightarrow} W\left(Z_{2}\right) \rightarrow 0
$$

is split exact.

Proof. Except for showing $\operatorname{Kernel}(\partial+\partial) \subseteq$ Image $(i)$, the remarks above about $i$ and the exact sequence $0 \rightarrow W(Z) \rightarrow W(Z(1 / 2)) \stackrel{\partial}{\longrightarrow} W\left(Z_{2}\right) \rightarrow 0$ of [5] make proving exactness trivial. But $\operatorname{Kernel}(\partial+\partial) \subseteq \operatorname{Image}(i)$ is also clear once we show that $(\langle\alpha\rangle,\langle\alpha\rangle) \in \operatorname{Image}(i)$, where $\langle\alpha\rangle=\left\langle 2 x^{2}-y\right\rangle$ is the generator of the torsion summand of $W\left(Z\left(\frac{1}{2}\right)\right)$. Now let $e_{1}$ and $e_{2}$ be the generators of a free $Z$-module with $e_{1} \cdot e_{1}=e_{2} \cdot e_{2}=0, e_{1} \cdot e_{2}=1, T\left(e_{1}\right)=e_{2}$ and $T\left(e_{2}\right)=e_{1}$. Set $I$ equal to the rank one submodule generated by $e_{1}+e_{2}$ and $I^{\perp}$ equal to the rank one submodule generated by $e_{1}-e_{2}$. Clearly, $\left(e_{1}+e_{2}\right) \cdot\left(e_{1}+e_{2}\right)=2$ and $\left(e_{1}-e_{2}\right) \cdot\left(e_{1}-e_{2}\right)=-2$. So $\langle I\rangle=\langle 1\rangle+\langle\alpha\rangle$ and $\left\langle I^{\perp}\right\rangle=-\langle 1\rangle+\langle\alpha\rangle$ in the image of $i$. Thus $(\langle\alpha\rangle,\langle\alpha\rangle)=(0,\langle 1\rangle)+(-\langle 1\rangle, 0)+(\langle 1\rangle+\langle\alpha\rangle,-\langle 1\rangle+\langle\alpha\rangle)$ is in the image of $i$. It is now easy to find a splitting map for $\partial+\partial$.

Corollary 2. $W_{0}\left(Z_{2} ; Z\right) \cong Z \oplus Z \oplus Z_{2}$. 
It is now useful to introduce the torsion index. We first observe that a pair $(X, Y)$ lies in the image of $i$ if and only if $X-\operatorname{sgn}(X)\langle 1\rangle=Y-\operatorname{sgn}(Y)\langle 1\rangle$. Now the torsion index is the homomorphism trs: $W_{0}\left(Z_{2} ; Z\right) \rightarrow Z_{2}$ given by the composition $W_{0}\left(Z_{2} ; Z\right) \rightarrow W(Z(1 / 2)) \stackrel{\partial}{\longrightarrow} W\left(Z_{2}\right)$ as $(T, V) \mapsto\left[I_{+} \otimes Z(1 / 2)\right] \stackrel{\partial}{\longrightarrow}$ $\partial\left[I_{+} \otimes Z(1 / 2)\right]$ or equivalently $(T, V) \mapsto\left[I_{-} \otimes Z(1 / 2)\right] \stackrel{\partial}{\longmapsto} \partial\left[I_{-} \otimes Z(1 / 2)\right]$.

Proposition 3. An element $\langle T, V\rangle$ in $W_{0}\left(Z_{2} ; Z\right)$ is uniquely determined by

(a) $\operatorname{sgn} V$,

(b) $\operatorname{sgn}\left(I_{+}\right)-\operatorname{sgn}\left(I_{-}\right)$,

(c) $\operatorname{trs}\langle T, V\rangle$.

Proof. Observe that sgn $V=\operatorname{sgn} I_{+}+\operatorname{sgn} I_{-}$and hence (a) and (b) determine the image of $i$ modulo the torsion in $W(Z(1 / 2)) \oplus W(Z(1 / 2))$. Now the remarks above about trs complete the proof.

It will be useful to us in $\$ 3$ to recount the well-known (cf. [7]) homomorphism $\partial^{\prime}: W(Q) \rightarrow W(Q / Z)$, where $W(Q / Z)$ is the Witt group of finite abelian groups with their inner products taking their values in $Q / Z$. So let $(M, \alpha)$ be an inner product space over $Q$. We form a lattice $L$ in $M$; that is, a finitely generated $Z$-submodule of $M$ such that $L=Z x_{1}+\ldots+Z x_{k} \subseteq M$, where the set $\left\{x_{1}, \ldots, x_{k}\right\}$ contains a basis for $M$ over $Q$. We further stipulate that the elements of $\left\{x_{1}, \ldots, x_{k}\right\}$ be chosen so that $\alpha\left(x_{i}, x_{j}\right) \in Z$. The dual lattice $L^{\#}$ is defined by setting $L^{\#}=\{r \in M \mid \alpha(r, L) \subseteq Z\}$. It is now easy to show that $M \supseteq L^{\#} \supseteq L$ and $L^{\#} \cong \operatorname{Hom}_{Z}(L, Z)$. We now define $\partial^{\prime}: W(Q) \rightarrow W(Q / Z)$ by $\partial^{\prime}(M, \alpha)=\left\langle L^{\#} / L\right\rangle$ where $L^{\#} / L$ has the inner product $\bar{\alpha}$ given for $x_{1}^{\#}, x_{2}^{\#} \in L^{\#}$ by $\bar{\alpha}\left(x_{1}^{\#}+L, x_{2}^{\#}+L\right)=\rho\left(\alpha\left(x_{1}^{\#}, x_{2}^{\#}\right)\right)$, where $\rho: Q \rightarrow Q / Z$ is the quotient homomorphism. We will use the easily verified fact that when $\partial^{\prime}$ is restricted to the image of $W(Z(1 / 2))$ in $W(Q)$ under inclusion, it agrees with $\partial: W(Z(1 / 2)) \rightarrow$ $W\left(Z_{2}\right)$.

2. Here we give a proof that ab: $O_{4}\left(Z_{2}\right)^{e} \rightarrow W_{0}\left(Z_{2} ; Z\right)$ is an isomorphism and cite a complete set of invariants for $\mathrm{O}_{4}\left(Z_{2}\right)^{e}$. This enables us to completely determine $\mathrm{O}_{4}\left(Z_{2}\right)$ as was indicated in the introduction.

From [12] we deduce an exact sequence

$$
0 \rightarrow \Omega_{4}\left(Z_{2}\right) \rightarrow 0_{4}\left(Z_{2}\right) \stackrel{\alpha}{\longrightarrow} A_{4} \stackrel{\partial}{\longrightarrow} \Omega_{3}\left(Z_{2}\right) \rightarrow 0,
$$

where $\Omega_{*}\left(Z_{2}\right)$ is the bordism ring of fixed point free orientation preserving smooth involutions. In [12] Rosenzweig shows that $\Omega_{n}\left(Z_{2}\right) \cong \Omega_{n} \oplus \mathcal{I}_{n-1}$; so $\Omega_{4}\left(Z_{2}\right) \cong Z$ and $\Omega_{3}\left(Z_{2}\right) \cong Z_{2}$. He also shows that

$$
\begin{aligned}
A_{4} \cong & \sum_{p+q=5} H_{p}\left(B O(0), B S O(0) ; \Omega_{q}\right) \oplus \sum_{p+q=3} H_{p}\left(B O(2), B S O(2) ; \Omega_{q}\right) \\
& \oplus \sum_{p+q=1} H_{p}\left(B O(4), B S O(4) ; \Omega_{q}\right) .
\end{aligned}
$$


It is easy to see that

and

$$
\sum_{p+q=5} H_{p}\left(B O(0), B S O(0) ; \Omega_{q}\right) \cong \Omega_{4}
$$

$$
\sum_{p+q=1} H_{p}\left(B O(4), B S O(4) ; \Omega_{q}\right) \cong H_{1}\left(B O(4), B S O(4) ; \Omega_{0}\right) .
$$

To calculate the latter group as well as the middle summand of $A_{4}$ we recall that the cohomology exact triangle

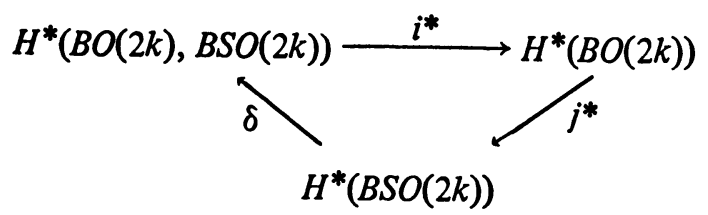

becomes

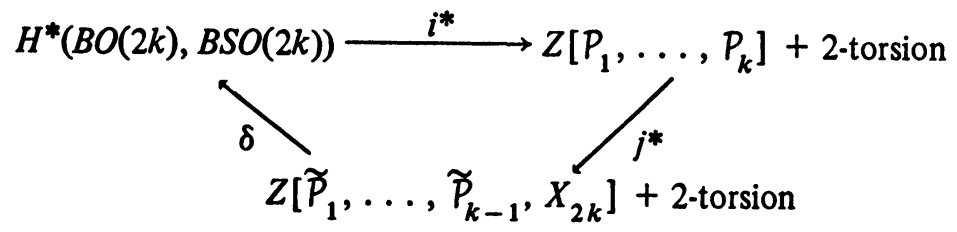

where $P_{m} \in H^{4 m}(B O(2 k))$ is the universal Pontrjagin class. Furthermore $j^{*}\left(P_{m}\right)$ $=\widetilde{P}_{m}$ for $1 \leqslant m \leqslant k-1, X_{2 k} \in H^{2 k}(B S O(2 k))$ is the Euler class, $j^{*}\left(P_{k}\right)=$ $X_{2 k}^{2}$ and $j^{*}$ maps the 2-torsion of $H^{*}(B O(2 k))$ onto the 2-torsion of $H^{*}(B S O(2 k))$. By the universal coefficient theorem $H_{1}(B O(4), B S O(4) ; Z) \cong$ torsion part of $H^{2}(B O(4), B S O(4) ; Z)$ and this is easily seen to be isomorphic to $Z_{2}$. It now remains to calculate $\Sigma_{p+q=3} H_{p}\left(B O(2), B S O(2) ; \Omega_{q}\right)$, which is isomorphic to $H_{3}(B O(2), B S O(2) ; Z)$. The free part of $H_{3}(B O(2), B S O(2) ; Z)$ clearly has rank one. Its torsion part is isomorphic to the torsion part of $H^{4}(B O(2), B S O(2) ; Z)$. Since $H^{3}(B S O(2))=0, H^{4}(B O(2)) \cong Z \oplus Z_{2}$ and $H^{4}(B S O(2)) \cong Z$, it follows that the torsion part of $H^{4}(B O(2), B S O(2) ; Z)$ is isomorphic to $Z_{2}$. We sum up that $A_{4} \cong Z \oplus Z \oplus Z_{2} \oplus Z_{2}$. The above exact sequence now becomes

$$
0 \rightarrow Z \rightarrow 0_{4}\left(Z_{2}\right) \stackrel{\alpha}{\longrightarrow} Z \oplus Z \oplus Z_{2} \oplus Z_{2} \stackrel{\partial}{\longrightarrow} Z_{2} \rightarrow 0 .
$$

Conner [4, (Theorem 4.8)] showed that any torsion class in $\Omega_{*}\left(Z_{2}\right)$ is the image under $\partial$ of an element of order 2 . It is easily shown $O_{4}\left(Z_{2}\right) \cong Z \oplus Z \oplus Z \oplus Z_{2}$ and therefore that $\mathrm{O}_{4}\left(Z_{2}\right)^{e} \cong Z \oplus Z \oplus Z_{2}$.

The Atiyah-Bott homomorphism ab: $O_{4 k}\left(Z_{2}\right) \rightarrow W_{0}\left(Z_{2} ; Z\right)$ is given by $\left(T, M^{4 k}\right) \mapsto\left\langle T^{*}, H^{2 k}\left(M^{4 k} ; Z\right) /\right.$ torsion $\rangle$. Our interest here is in ab $10_{4 k}\left(Z_{2}\right)^{e}$ which we also denote by $a b$.

THEOREM 4. ab: $O_{4}\left(Z_{2}\right)^{e} \rightarrow W_{0}\left(Z_{2} ; Z\right)$ is an isomorphism. 
Proof. By our calculations of $\mathrm{O}_{4}\left(Z_{2}\right)^{e}$ and $W_{0}\left(Z_{2} ; Z\right)$, it suffices to show that $\mathrm{ab}$ is an epimorphism. This is easily seen (compare the proof of Proposition 1) by looking at the image under ab of $[T, C P(2)],[C, C P(2)]$ and $[C, C P(2)]-[T, C P(2)]+[d, C P(1) \times C P(1)]$, where $T\left[Z_{1}, Z_{2}, Z_{3}\right]=$ $\left[-Z_{1}, Z_{2}, Z_{3}\right], C\left[Z_{1}, Z_{2}, Z_{3}\right]=\left[\bar{Z}_{1}, \bar{Z}_{2}, \bar{Z}_{3}\right]$ and $d(x, y)=(y, x)$.

We now observe that $\mathrm{O}_{4}\left(Z_{2}\right)^{e}$ is completely determined by standard invariants. First we note that (in the terminology of Proposition 3) $\operatorname{sgn}\left(I_{+}\right)-\operatorname{sgn}\left(I_{-}\right)$ is equal up to sign to $\operatorname{sgn}(F \circ F)$, where $F$ is the fixed point set of the manifold in question (cf. [4]). Alexander has shown [1] that

$$
\operatorname{trs}\left\langle T^{*}, H^{2}\left(M^{4} ; Z\right) / \text { torsion }\right\rangle \equiv \chi_{0}(F)+1 / 2\left(\operatorname{sgn}\left(M^{4}\right)+\operatorname{sgn}(F \circ F)\right)(\bmod 2),
$$

where $\chi_{0}(F)$ is the sum of the Euler characteristics of components of $F$ with dimensions divisible by four. Summing up, we get the next proposition from Proposition 3.

THEOREM 5. The class of $\left(T, M^{4}\right)$ in $\mathrm{O}_{4}\left(Z_{2}\right)^{e}$ is completely determined by

(i) $\operatorname{sgn}\left(M^{4}\right)$,

(ii) $\operatorname{sgn}(F \circ F)$,

(iii) $\chi_{0}(F)(\bmod 2)$.

It follows from the above that any representative of the torsion class in $\mathrm{O}_{4}\left(Z_{2}\right)$ must have an odd number of isolated fixed points.

Finally, we apply Theorem 4 to show that a 4 manifold with involution is bordant to a manifold with involution which has no unoriented components in its fixed point set if and only if the Euler characteristic of the two dimensional components of its fixed point set is even. The following example shows that this result is false in other dimensions divisible by four. Namely, take $C P(4) \cup C P(2) \times C P(2)$ with the involution conjugation $\cup$ (conjugation $\times$ conjugation). The fixed point set is $R P(4) \cup R P(2) \times R P(2)$ which vacuously has $\chi_{2} \equiv 0(\bmod 2)$, but $w_{1}^{4} \neq 0$. Nothing bordant to this can have an oriented fixed point set. Cross with $C P(2 k)$ with conjugation to get the other dimensions.

THEOREM 6. Given $\left(T, M^{4 k}\right)$ with $\chi_{2}(F) \equiv 0(\bmod 2)$ there exists $\left(T_{1}, N^{4 k}\right)$ such that $\mathrm{ab}\left(T, M^{4 k}\right)=\mathrm{ab}\left(T_{1}, N^{4 k}\right)$ and $\left(T_{1}, N^{4 k}\right)$ has no unoriented components in its fixed point set. So

Proof. Let $\chi_{2}(F) \equiv 0(\bmod 2)$. This implies that $\chi(M) \equiv \chi_{0}(F)(\bmod 2)$

$$
\begin{aligned}
\operatorname{trs} & \equiv \chi(M)+(1 / 2)(\operatorname{sgn} M+\operatorname{sgn} F \circ F) \quad(\bmod 2) \\
& \equiv \operatorname{sgn} M+(1 / 2)(\operatorname{sgn} M+\operatorname{sgn} F \circ F)(\bmod 2) \\
& \equiv(3 / 2) \operatorname{sgn} M+(1 / 2) \operatorname{sgn} F \circ F \quad(\bmod 2) \\
& \equiv(3 / 2)\left(\operatorname{sgn} I_{+}+\operatorname{sgn} I_{-}\right)+(1 / 2)\left(\operatorname{sgn} I_{+}-\operatorname{sgn} I_{-}\right)(\bmod 2) \\
& \equiv \operatorname{sgn} I_{-} \quad(\bmod 2) .
\end{aligned}
$$


Hence, only certain classes in $W_{0}\left(Z_{2} ; Z\right)$ may occur if $\chi_{2}(F) \equiv 0(\bmod 2)$. In fact, only classes of the form $(m, 2 n, 0)$ and $(m, 2 n+1,1)$ may occur and they are represented by copies of $(T, C P(2 n))$ and $(d, C P(n) \times C P(n))$.

COROLlaRy 7. A bordism class in $\mathrm{O}_{4}\left(Z_{2}\right)$ has a representative with no unoriented components in its fixed point set if and only if $\chi_{2}(F) \equiv 0(\bmod 2)$.

Proof. First note that $\chi_{2}(F)(\bmod 2)$ is a bordism invariant. Then combine the corollary of [1] with our Theorems 4 and 6 .

3. We now show how to use the integer representation of an involution $\left(T^{*}, H^{2 k}\left(M^{4 k} ; Z\right) /\right.$ torsion $)$ on $H^{2 k}\left(M^{4 k} ; Z\right) /$ torsion to determine the invariants trs, $\chi_{0}(F)(\bmod 2)$ and $\chi_{2}(F)(\bmod 2)$.

According to Reiner (cf. [6]) any involution $T$ on a free $Z$-module $V$ splits $V$ into indecomposable summands of the form $Z \oplus \ldots \oplus Z \oplus Z\left(Z_{2}\right) \oplus \ldots \oplus$ $Z\left(Z_{2}\right) \oplus \widetilde{Z} \oplus \ldots \oplus \widetilde{Z}$, where $T$ is fixed on $Z$ summands, $T(1)=-1$ on the $\widetilde{Z}$ summands and each $Z\left(Z_{2}\right)$ summand has rank two with $T$ interchanging the generators. We will be interested in the numbers modulo 2 of copies of $Z, \widetilde{Z}$ and $Z\left(Z_{2}\right)$ which appear in the representation.

Recall [5] that $q(T, V)$ is defined to be $w(\beta)-\operatorname{sgn} w(\beta)<1\rangle \in W(Q)$, where $\beta$ is obtained by inducing from the inner product on $V$ an inner product on $I_{+}$ $\otimes Q$ and setting $\beta(v, w)=1 / 2(v, w)$ for all $v, w \in I_{+} \otimes Q$. Since $w(\beta)$ lies in $W(Z(1 / 2))$, we may as well read this difference in $W(Z(1 / 2))$. Now the fact that sgn splits the exact sequence $0 \rightarrow W(Z) \rightarrow W(Z(1 / 2)) \stackrel{\partial}{\longrightarrow} W\left(Z_{2}\right) \rightarrow 0$ allows us to interpret $q(T, V)$ as $\partial(w(\beta)) \in W\left(Z_{2}\right)$. To find $\partial(w(\beta))$ we choose the lattice

$$
L=Z x_{1}+\ldots+Z x_{k}+Z\left(y_{1}+T y_{1}\right)+\ldots+Z\left(y_{n}+T y_{n}\right)
$$

where the elements $x_{1}, \ldots, x_{k}$ are generators of all the $Z$ summands in $V$ and the $y_{1}, \ldots, y_{n}$ represent all the copies of $Z\left(Z_{2}\right)$. This choice of $L$ is a lattice in $I_{+}$. Now consider the diagram

$$
I_{+} \stackrel{h}{\cong} \operatorname{Hom}_{Z}(L, Z)
$$

where the maps are given by the following:

$$
\begin{aligned}
x \text { fixed, } x \longmapsto & (x,-), \\
T y \neq \pm y, T y+y \longmapsto & 1 / 2(T y+y,-) \\
& \begin{array}{cc}
1 / 2(x,-) & 1 / 2(T y+y) \\
\uparrow_{x} & T y+y
\end{array}
\end{aligned}
$$

By comparing the image of the embedding of the lattice $L$ (via $\beta$ ) in its dual 
lattice $\operatorname{Hom}_{Z}(L, Z)$ with that of $I_{+}$, we note that the rank of $L^{\#} / L$ as a $Z_{2}$-vector space is equal to the number of copies of $Z$ in the representation of $V$. Now the next theorem follows from the fact that the class of any inner product space in $W\left(Z_{2}\right)$ is determined by its rank modulo 2 .

THEOREM 8. If $r$ is the number of copies of $Z$ occurring in the integer representation of $(T, V)$, then $q(T, V) \equiv r(\bmod 2)$.

REMARK. It is seen by a straightforward argument that $q(T, V)=$ $\left\langle H_{1}\left(Z_{2} ; V\right)\right\rangle \in W\left(Z_{2}\right)$. It can also be proved that $\operatorname{dim}_{Z_{2}}\left(H_{2}\left(Z_{2} ; V\right)\right) \equiv t(\bmod 2)$.

Given $\left(T^{*}, H^{2 k}\left(M^{4 k} ; Z\right) /\right.$ torsion $)$, Alexander [1] showed that

$$
\left.q\left(T^{*}, H^{2 k} ; Z\right) / \text { torsion }\right) \equiv \chi_{0}(F)(\bmod 2)
$$

leading to the next corollary.

Corollary 9. $\chi_{0}(F) \equiv r(\bmod 2)$.

THEOREM 10. The number of copies of $Z\left(Z_{2}\right)$ occurring in the integer representation of $(T, V)$ is congruent to trs modulo 2.

PRoof. Recall that trs was given by restricting the inner product on $V$ to $I_{+}$, extending it to $I_{+} \otimes Z(1 / 2) \in W(Z(1 / 2))$ and then applying $\partial$. By referring to diagram (1), it is easy to see that under the new embedding of $L$ into $\operatorname{Hom}_{Z}(L, Z), L^{\#} / L$ has rank as a $Z_{2}$-vector space equal to the number of copies of $Z\left(Z_{2}\right)$ in the representation of $(T, V)$. The argument is then the same as that of Theorem 8.

Let $t$ be equal to the number of copies of $\widetilde{Z}$ occurring in the representation of $\left(T^{*}, H^{2 k}\left(M^{4 k} ; Z\right) /\right.$ torsion $)$. In the proof of our next theorem, it will be necessary for us to use the fact that $\chi(F)=\chi_{0}(F)+\chi_{2}(F)$, where $\chi_{2}(F)$ is, an. alogously to $\chi_{0}(F)$, defined to be the sum of the Euler characteristics of those components of $F$ with dimensions congruent to 2 modulo 4. This is because orientation preserving involutions on even dimensional manifolds do not have odd dimensional components in their fixed point sets.

THEOREM $11 . \chi_{2}(F) \equiv t(\bmod 2)$.

Proof. By Poincaré duality,

$$
\chi\left(M^{4 k}\right) \equiv \operatorname{rank} H^{2 k}\left(M^{4 k} ; Q\right) \quad(\bmod 2) .
$$

Now, since the rank of each copy of $Z\left(Z_{2}\right)$ in the representation of $\left(T^{*}, H^{2 k} ;\left(M^{4 k} ; Z\right) /\right.$ torsion $)$ is two, it follows that

$$
\chi\left(M^{4 k}\right) \equiv r+t(\bmod 2) .
$$

The theorem follows from the fact that

$$
\chi\left(M^{4 k}\right) \equiv \chi_{0}(F)+\chi_{2}(F) \equiv r+\chi_{2}(F)(\bmod 2),
$$

where the first congruence is a known result from Smith theory (cf. [3]). 
4. In this section we establish the fact that in the case of a $(4 n+2)$-dimensional closed oriented differentiable manifold with orientation preserving differentiable involution, $\chi_{0}(F)$ and $\chi_{2}(F)$ are both even. It is easy to construct examples showing that this result cannot be improved upon.

The next lemma is crucial to proving the above. We recall that for $N^{2 r+1}$ a closed orientable manifold, the semicharacteristic of $N^{2 r+1}$ with respect to a coefficient field $F$ is defined to be

$$
\sigma\left(N^{2 r+1} ; F\right)=\sum_{i=0}^{r} \operatorname{dim} H_{i}\left(N^{2 r+1} ; F\right)
$$

reduced modulo 2 .

LEMMA 12. If $B^{4 n+2}$ is a compact manifold with boundary, then

$$
\chi\left(B^{4 n+2}\right) \equiv \sigma\left(\partial B^{4 n+2} ; Q\right)(\bmod 2) .
$$

Proof. Consider the following segment of the long exact sequence of a pair with rational coefficients

$$
\ldots \stackrel{\delta_{i}}{\longrightarrow} H^{i}(B, \partial B) \stackrel{\beta_{i}}{\longrightarrow} H^{i}(B) \stackrel{\alpha_{i}}{\longrightarrow} H^{i}(\partial B) \stackrel{\delta_{i+1}}{\longrightarrow} H^{i+1}(B, \partial B) \rightarrow \ldots
$$

We observe that

$$
\operatorname{dim}\left(H^{i}(B)\right)=\operatorname{dim}\left(\operatorname{Image} \beta_{i}\right)+\operatorname{dim}\left(\operatorname{Image} \alpha_{i}\right)
$$

and

$$
\operatorname{dim}\left(H^{i-1}(\partial B)\right)=\operatorname{dim}\left(\operatorname{Image} \alpha_{i-1}\right)+\operatorname{dim}\left(\operatorname{Image} \delta_{i}\right) .
$$

It is easy to reduce these two equations to

$$
\begin{aligned}
\operatorname{dim}\left(H^{i}(B)\right)+ & \operatorname{dim}\left(H^{4 n+2-i}(B)\right)+H^{i-1}(\partial B) \\
& =\operatorname{dim}\left(\text { Image } \alpha_{i}\right)+\operatorname{dim}\left(\text { Image } \alpha_{i-1}\right)
\end{aligned}
$$

It follows by summing this equation from $i=1$ to $2 n+1$ that

$$
\chi(B)+\operatorname{dim}\left(H^{2 n+1}(B)\right)+\sigma(\partial B ; Q) \equiv \operatorname{dim}\left(I m a g e \alpha_{2 n+1}\right)(\bmod 2) .
$$

Now the fact that $\operatorname{dim}\left(H^{2 n+1}(B)\right)=\operatorname{dim}\left(\operatorname{Image} \alpha_{2 n+1}\right)+\operatorname{dim}\left(\operatorname{Image} \beta_{2 n+1}\right)$ implies that

$$
\chi(B)+\sigma(\partial B ; Q) \equiv \operatorname{dim}\left(\operatorname{Image} \beta_{2 n+1}\right)(\bmod 2) .
$$

But there is a nonsingular antisymmetric bilinear form induced on the image of

$$
\beta_{2 n+1}^{*}: H^{2 n+1}\left(B^{4 n+2}, \partial B^{4 n+2} ; Q\right) \rightarrow H^{2 n+1}\left(B^{4 n+2} ; Q\right)
$$

and this shows that the image of $\beta_{2 n+1}^{*}$ has even dimension. The proof is now complete.

We write our closed oriented differentiable $(4 n+2)$-manifold $M^{4 n+2}$ with 
orientation preserving differentiable involution $T$ as $M^{4 n+2}=W^{4 n+2} \cup N$ where $N$ is the normal bundle to the fixed point set and $W^{4 n+2}$ and $N$ share a common boundary. Now the additivity of the Euler characteristic implies $\chi\left(M^{4 n+2}\right)=$ $\chi\left(W^{4 n+2}\right)+\chi(F)$. It follows from Smith theory (cf. [3]) that $\chi\left(M^{4 n+2}\right)+$ $\chi(F)=2 \chi\left(M^{4 n+2} / T\right)$. Since $M^{4 n+2} / T$ is a rational homology manifold, the usual argument yielding $2 \mid \chi\left(M^{4 n+2}\right)$ applies to show that $2 \mid \chi\left(M^{4 n+2} / T\right)$. The fact that $2 \mid \chi\left(M^{4 n+2}\right)$ implies that $\chi\left(M^{4 n+2}\right) \equiv-\chi\left(M^{4 n+2}\right)(\bmod 4)$ and consequently $\chi\left(M^{4 n+2}\right)=\chi(F)(\bmod 4)$ from which it follows that $4 \mid \chi\left(W^{4 n+2}\right)$. Again from Smith theory $\chi\left(W^{4 n+2}\right)=2 \chi\left(W^{4 n+2} / T\right)$ which shows that $2 \mid \chi\left(W^{4 n+2} / T\right)$. We are now prepared to prove our theorem.

THEOREM 13. Let $M^{4 n+2}$ be a closed oriented differentiable manifold with orientation preserving differentiable involution. Then $\chi_{0}(F) \equiv \chi_{2}(F) \equiv$ $0(\bmod 2)$.

Proof. Since $\chi\left(M^{4 n+2}\right) \equiv \chi(F) \equiv 0(\bmod 2)$, the theorem will follow from the above remarks by showing that $\chi\left(W^{4 n+2} / T\right) \equiv \chi_{0}(F)(\bmod 2)$. So we first observe that

$$
\chi(W / T) \equiv \sigma(\partial W / T ; Q)=\sigma(\partial N / T ; Q)=\sigma\left(\partial N / T ; Z_{2}\right)(\bmod 2)
$$

where the last equality follows from [9]. Now by the Leray-Hirsch theorem $\partial N / T$ has the $Z_{2}$-cohomology of a union of copies of $F^{4 l} \times \mathrm{RP}(4 m+1)$ and $F^{4 l+2} \times \mathbf{R} P(4 m+3)$. Recall that

$$
\sigma\left(F^{2 k} \times \mathbf{R} P(2 m+1) ; Z_{2}\right) \equiv \sigma\left(\mathbf{R} P(2 m+1) \chi\left(F^{2 k}\right)\right)(\bmod 2)
$$

[9]. This shows that $\sigma\left(\partial N / T ; Z_{2}\right) \equiv \chi_{0}(F)(\bmod 2)$ and completes the proof.

5 . We now consider those elements of $W_{0}\left(Z_{2} ; Z\right)$ which admit a representative of type II. The ideal of such elements will be denoted by $E_{0}\left(Z_{2} ; Z\right)$. We recall that an element $(T, V)$ in $W_{0}\left(Z_{2} ; Z\right) \cong Z \oplus Z \oplus Z_{2}$ can be written as ( $\operatorname{sgn} V$, sgn $I_{+}-\operatorname{sgn} I_{-}$, trs). Our initial observation is that a single copy of the group ring $Z\left(Z_{2}\right)$ with generators $x$ and $y$ where $(x, x)=(y, y)=0$ and $(x, y)=$ 1 represents the element $(0,2,1) \in E_{0}\left(Z_{2} ; Z\right)$. It is well known that elements of the form $(8 m, 0,0)$ also occur in $E_{0}\left(Z_{2} ; Z\right)$. This leads to our next theorem.

THEOREM 14. The ideal $E_{0}\left(Z_{2} ; Z\right)$ is generated additively by elements of the form $(8 m, 0,0)$ and $(0,2,1)$.

Proof. The proof is by elimination of the other possibilities. First observe that elements of the form $(8 m, 2 k+1,1)$ and $(8 m, 2 k+1,0)$ are not in $E_{0}\left(Z_{2} ; Z\right)$ since sgn $I_{+}+\operatorname{sgn} I_{-}=8 m$ and $\operatorname{sgn} I_{+}-\operatorname{sgn} I_{-}=2 k+1$ implies 2 sgn $I_{+}=8 m+2 k+1$ which is impossible. Furthermore, $(0,2,0)$ is not in $E_{0}\left(Z_{2} ; Z\right)$. To see this, recall that $(0,2,0)$ written in terms of $\left(\operatorname{sgn} I_{+}, \operatorname{sgn} I_{-}\right.$, 
trs $)$ is $(1,-1,0)$ which has its image under $i: W_{0}\left(Z_{2} ; Z\right) \rightarrow W(Z(1 / 2)) \oplus W(Z(1 / 2))$ in $W(Z) \oplus W(Z)$. Now observe that for $(1,-1,0)$ to admit a representative of type II, it must do so in each summand independently. But neither summand has signature divisible by 8 and hence $(1,-1,0)$ is not of type II. Having eliminated $(0,2,0)$ automatically eliminates the rest of the possibilities and the theorem follows.

The techniques of the next proof readily yield that $q[(8 m, 0,0)]=$ $q[(0,2,1)]=0$ implying that $q\left(E_{0}\left(Z_{2} ; Z\right)\right)=\{0\}$. Thus $q$ induces a homomorphism on the quotient ring $W_{0}\left(Z_{2} ; Z\right) / E_{0}\left(Z_{2} ; Z\right)$.

THEOREM 15 . $W_{0}\left(Z_{2} ; Z\right) / E_{0}\left(Z_{2} ; Z\right) \cong Z_{8} \oplus Z_{2}$ where the summands are given by $(\operatorname{sgn} V(\bmod 8), q(T, V))$.

Proof. From Theorem 14, we note that $(0,0,1)$ is a representative of the generator of the $Z_{2}$-summand. But in this representative sgn $I_{+}+\operatorname{sgn} I_{-}=$ 0 and $\operatorname{sgn} I_{+}-\operatorname{sgn} I_{-}=0$ yielding sgn $I_{+}=\operatorname{sgn} I_{-}=0$. The fact that $\operatorname{sgn} I_{+}=$ 0 means that when we select an orthogonal basis for $I_{+} \otimes Z(1 / 2)$ (cf. $[11$, p. 6]), say $\left\langle u_{1}\right\rangle \oplus \ldots \oplus\left\langle u_{n}\right\rangle, n$ must be an even number. We can further select the $u_{i}$ so that $\left(u_{i}, u_{i}\right)$ is equal to either 2 or 1 in $Z(1 / 2)$. Now, by noting that trs is the image in $W\left(Z_{2}\right)$ of this form under the boundary homomorphism and $q(T, V)$ is the image under the boundary homomorphism of $1 / 2$ times this form, one can check that trs is given by the number modulo 2 of elements $u_{i}$ in the above designed basis with $\left(u_{i}, u_{i}\right)=2$, and similarly $q(T, V)$ is given by the number modulo 2 of basis elements $u_{j}$ with $\left(u_{j}, u_{j}\right)=1$. Since $n$ is even, it follows that trs is equal to $q(0,0,1)$ and the theorem then follows from Theorem 14.

We now determine the multiplicative structure of $W_{0}\left(Z_{2} ; Z\right) / E_{0}\left(Z_{2} ; Z\right)$. The task is, of course, to determine $q\left((T, V)\left(T_{1}, V_{1}\right)\right)$. To do so we apply Theorem 8 to the Reiner decomposition of $\left(T \otimes T_{1}, V \otimes V_{1}\right)$. The result is that

$$
\begin{aligned}
q\left(T \otimes T_{1}, V \otimes V_{1}\right) & \equiv q(T, V) q\left(T_{1}, V_{1}\right) \\
& +[\operatorname{sgn} V-q(T, V)]\left[\operatorname{sgn} V_{1}-q\left(T, V_{1}\right)\right] \quad(\bmod 2) .
\end{aligned}
$$

The terms in the last product occur because the number of copies of $Z$ plus the number of copies of $\widetilde{Z}$ in the Reiner decomposition of $(T, V)$ are congruent to sgn $V$ modulo 2. The next theorem follows from the above discussion.

THEOREM 16. The multiplication in $W_{0}\left(Z_{2} ; Z\right) / E_{0}\left(Z_{2} ; Z\right)$ is given by

$(\operatorname{sgn} V(\bmod 8), q(T, V)) \cdot\left(\operatorname{sgn} V_{1}(\bmod 8), q\left(T_{1}, V_{1}\right)\right)$

$$
\begin{aligned}
=\left(\operatorname{sgn} V \operatorname{sgn} V_{1}\right. & (\bmod 8), \operatorname{sgn} V \operatorname{sgn} V_{1} \\
& \left.+\operatorname{sgn} V q\left(T_{1}, V_{1}\right)+\operatorname{sgn} V_{1} q(T, V)\right)
\end{aligned}
$$

where the last summand is read modulo 2. 


\section{BIBLIOGRAPHY}

1. J. P. Alexander, $A W\left(Z_{2}\right)$ Invariant for orientation preserving involutions, Proc. Amer. Math. Soc. 51 (1975), 455-460.

2. J. P. Alexander, P. E. Conner, G. C. Hamrick and J. W. Vick, Witt classes of integral representations of an abelain p-group, Bull. Amer. Math. Soc. 80 (1974), 1179-1182.

3. G. E. Bredon, Introduction to compact transformation groups, Academic Press, New York, 1972.

4. P. E. Conner, Lectures on the action of a finite group, Lecture Notes in Math., no. 73, Springer-Verlag, Berlin and New York, 1968. MR 41 \#2670.

5. P. E. Conner and F. Raymond, $A$ quadratic form on the quotient of a periodic map, Semigroup Forum 7 (1974).

6. C. W. Curtis and I. Reiner, Representation theory of finite groups and associative algebras, Pure and Appl. Math., vol. 11, Interscience, New York, 1962. MR 26 \#2519.

7. J. P. Alexander, G. C. Hamrick and J. W. Vick, Bilinear forms and cyclic group actions, Bull. Amer. Math. Soc. 80 (1974), 730-734.

8. K. Jänich and E. Ossa, On the signature of an involution, Topology 8 (1969), 27-30. MR 38 \#6613.

9. G. Lusztig, J. Milnor and F. P. Peterson, Semi-characteristics and cobordism, Topology 8 (1969), 357-359. MR 39 \#7612.

10. J. W. Milnor, On simply connected 4-manifolds, Sympos. Internacional de Topologia Algebraica [Internat. Sympos. on Algebraic Topology], Universidad Nacional Autónoma de México and UNESCO, Mexico City, 1958. MR 21 \#2240.

11. J. W. Milnor and D. Husemoller, Symmetric bilinear forms, Springer-Verlag, New York, 1973.

12. H. Rosenzweig, Bordism of involutions on manifolds, Illinois J. Math. 16 (1972), 1-10. MR 44 \#7568.

DEPARTMENT OF MATHEMATICS, LOUISIANA STATE UNIVERSITY, BATON ROUGE, LOUISIANA 70803 